\title{
The Effectiveness of a Nursing Care Program Supplemented with Iron Intake on Anemia and Fatigue in Cancer Patients Undergoing Chemotherapy
}

\author{
Zeinab Barahoui ${ }^{1}$, Fatemeh Kiyani ${ }^{2,{ }^{*}}$, Tayebe Lashkari ${ }^{3}$ and Mojtaba Tasbandi ${ }^{4}$ \\ ${ }^{1}$ Department of Nursing, Faculty of Nursing and Midwifery, Zahedan University of Medical Sciences, Zahedan, Iran \\ ${ }^{2}$ Community Nursing Research Center, Zahedan University of Medical Sciences, Zahedan, Iran \\ ${ }^{3}$ Master of Emergency Nursing, Khatam Hospital, Zahedan, Iran \\ ${ }^{4}$ Radiation Oncology and Head Manager of Department of Radiation Oncology, University of Zahedan Medical School, Zahedan, Iran \\ "Corresponding author: Faculty of Nursing and Midwifery, Zahedan University of Medical Sciences, Zahedan, Iran. Tel: +98-5433442481, Email: fkiani2011@yahoo.com
}

Received 2020 November 21; Accepted 2020 November 21

\begin{abstract}
Background: Fatigue is one of the most common complications of cancer. Anemia can be one of the most prevalent causes of fatigue in cancer patients.

Objectives: The present study aimed to explore the effectiveness of a nursing care program supplemented with iron intake on the anemia and fatigue in cancer patients undergoing chemotherapy.

Methods: The present study was conducted in a quasi-experimental design on two groups of 90 cancer patients in selected hospitals. The participants were selected via convenience sampling and were randomly assigned to the control and intervention groups. Before the intervention, the Functional Assessment of Cancer Therapy - Anemia (FACT-An) was completed by the patients in both groups. The patients in the control group did not receive any special care other than receiving $150 \mathrm{mg}$ of iron daily. However, the patients in the intervention group received $150 \mathrm{mg}$ of iron daily and attended a nursing care program with four individual training sessions (one session per week). Follow-up was performed for three weeks, and the questionnaire was completed again by the participants in both groups. The collected data were analyzed using the independent samples t-test, paired-samples t-test, and chi-square test in SPSS software (version 22). The data analysis process was performed at a significance level of less than 0.05 .

Results: The mean scores for the patient hemoglobin in the intervention and control groups after the intervention were $11.84 \pm$ 0.58 and $11.10 \pm 67.29$, showing a significant increase in favor of the intervention group $(\mathrm{P}<0.001)$. Furthermore, the results of the independent samples t-test showed that the mean score of fatigue for the patients in the intervention group (29.37 \pm 9.56$)$ was significantly lower than that of the participants in the control group $(47.55 \pm 11.22)(\mathrm{P}<0.001)$.

Conclusions: The present study showed that the implementation of a nursing care program for cancer patients receiving iron supplementation positively affects iron-related blood parameters and fatigue among these patients. Therefore, to control cancer patients' fatigue, medical staff must pay special attention to these training and care programs supplemented with iron intake.
\end{abstract}

Keywords: Fatigue, Cancer, Anemia, Care Plan Nursing

\section{Background}

Cancer is one of the most severe diseases of the present century and the second leading cause of death (1) after cardiovascular diseases (2). It is regarded as the third leading cause of death following cardiovascular diseases and accidents in Iran (3). Over 30,000 people die annually due to cancer (4). It is predicted that by 2030 , more than 13.1 million deaths in the world will be because of cancer (5).

Depending on the stage of cancer and the medical history of the patient, there are different types of treatment for cancer, such as surgery, radiotherapy, chemotherapy, or hormone therapy. Although these treatments may contribute to the survival of the patient, they may generate various complications $(4,6)$, among which fatigue is the most annoying (7-10), Reported in $70 \%$ to $100 \%$ of treated patients (4), fatigue is one of the most essential and definitive diagnoses of nursing. According to the North American Nursing Diagnosis (NANDA), fatigue refers to "a state of general, persistent feeling of boredom and reduction of physical and mental ability to lower than the normal level" (11). Burgess pointed out that the fatigue experienced by people with cancer is different from the fatigue experienced by healthy people and that these patients ex- 
perience more intense and sustained fatigue, which does not improve with adequate sleep and rest. Besides, 78 to $96 \%$ of cancer patients and 75 to $99 \%$ of patients undergoing chemotherapy experience symptoms of fatigue $(12,13)$. One of the essential factors affecting fatigue is anemia (14).

Iron deficiency anemia is prevalent in cancer patients (15). Chemotherapy can destroy the body's natural tissues causing bone marrow damage and can result in anemia, which leads to fatigue $(16,17)$. Chemotherapy-induced anemia (CIA) occurs when chemotherapy rapidly attacks dividing cells, including red blood cells (RBCs), and prevents them from dividing (18). Some studies have estimated the prevalence of anemia in patients undergoing chemotherapy to be $100 \%$ (19). For instance, Cellafound that $25 \%$ of cancer patients with hemoglobin less than 12 $\mathrm{mg} / \mathrm{dl}$ had difficulty performing their duties due to fatigue (20). Another study by Holzner (2002) showed that a decrease in blood hemoglobin levels is associated with an increase in the severity of fatigue in patients undergoing chemotherapy (21). Besides, Zeighami Mohammadi (2011) examined the relationship between anemia, the severity of fatigue, and quality of life in cancer patients undergoing chemotherapy and reported the prevalence of anemia to be $63.6 \%$ (19).

However, given the prevalence of iron deficiency, it is not often evaluated in cancer patients (22). One of the essential interventions in the treatment of anemia is iron supplementation (23). Nevertheless, despite the consumption of iron, it seems that its absorption in cancer patients is not enough. Therefore, experts point out that one of the most essential tasks of medical staff, especially nurses, is to diagnose anemia in cancer patients and eliminate its consequences, including fatigue, through unique care strategies (24).

It seems that a set of strategies should be considered to help these patients. Implementing training programs for cancer patients can reduce fatigue and other complications (25). Hence, teaching some practical strategies to these patients to reduce fatigue, to cope with the experience of the disease, and to overcome the pathological aspects of diagnosis, treatment, and follow-up courses is essential. In many studies, care planning focuses on patients' physical problems while many researchers believe that medical personnel, including nurses, should be trained in how to effectively do exercises and, in general, sports counseling and physical activity as part of routine examinations and procedures taken for patients including cancer patients (26). Some studies have shown that aerobic exercise improves erythrocyte counts, especially hemoglobin, during treatment and after chemotherapy (27). Some researchers have reported that taking iron supplements and aerobic exercises increase iron levels, ferritin, red blood cells, hemoglobin, hematocrit, and transferrin (28).

\section{Objectives}

Considering that planning a care program with an emphasis on training iron absorption strategies and its effect on fatigue control is a non-pharmacological, non-invasive, and low-cost method for controlling physical and psychological problems, the present study aims to determine the effectiveness of a nursing care program supplemented with iron intake on fatigue in cancer patients undergoing chemotherapy.

\section{Methods}

This study employed a quasi-experimental method with two groups and a pretest-posttest design that was conducted in 2020. The research project was approved by the Vice-Chancellor for Research and Technology of Zahedan University of Medical Sciences and by the Ethics Committee of the university under the ethics code IR.zaums.REC.1398.391. Following similar studies, the sample size was determined as 45 patients in each group with a $95 \%$ confidence interval, $90 \%$ statistical test power, and considering the possible dropout:

$$
\begin{aligned}
n= & \frac{\left(Z_{1-\frac{\alpha}{2}}+Z_{1-\beta}\right)^{2}\left(S_{1}^{2}+S_{2}^{2}\right)}{\left(\bar{X}_{1}-\bar{X}_{2}\right)^{2}} \\
= & 40.69 \\
& Z_{1-\frac{\alpha}{2}}=1.69 \mathrm{~S}_{1}=2.47 \bar{X}_{1}=0.34 \\
& Z_{1-\beta}=1.28 \mathrm{~S}_{2}=2.14 \bar{X}_{2}=2.00
\end{aligned}
$$

The participants in this study were 90 cancer patients undergoing chemotherapy in the oncology wards of University teaching hospitals in southeastern Iran. The patients were selected using convenience sampling based on the inclusion criteria (being at the age of 20 to 60 years, undergoing at least three courses of chemotherapy, having hemoglobin between 10 up to $12 \mathrm{~g} / \mathrm{dL}$, and having albumin of $3.5 \mathrm{~g} / \mathrm{dL}$ and above) and the exclusion criteria (noncompliance with medication schedule and patient death). For random allocation, first, the envelopes containing the group name cards were prepared for the total number of participants and randomly arranged. After selecting a participant, one of these cards was assigned to him/her specifying his/her group. Upon the random allocation, patients were divided into control and intervention groups. Before starting the study, the researcher provided the necessary explanations about the study to the patients and obtained their informed consent to participate in the study. 
The data were collected using the Patient Demographic and Clinical Information Questionnaire and the Functional Assessment of Cancer Therapy - Anemia (FACT-An). The demographic information questionnaire measured the participants' age, sex, occupation, education, duration of cancer, hemoglobin, hematocrit, RBC, and albumin. The FACT-An contains 13 statements related to fatigue (FACT-F) and 7 items on other anemia symptoms that are scored from 0 to 4 on a 5-point Likert scale. The total score on this scale ranges from 0 to 80 . The reliability of the instrument was confirmed abroad using Cronbach's alpha with $r=0.93$ and $r=0.95$, respectively. Moreover, the reliability and validity of this instrument were estimated by Ghaffari et al. using Cronbach's alpha method $(r=0.89)(24)$. Before the intervention, the FACT-An was completed by the participants in two groups to measure anemia-related fatigue. The patients in the control group did not receive any special care except for receiving $150 \mathrm{mg}$ of iron daily. The participants in the intervention group attended a nursing care program (iron intake, aerobic exercise, proper nutrition training) implemented in the form of four training sessions (one session per week) complemented with an iron intake of $150 \mathrm{mg}$ daily (according to the advice of an oncologist). Upon completing the 4 training sessions, the patients received an exercise recording checklist, a booklet, and a CD. Since the patients had regular visits to receive treatment regimens, face-to-face follow-up was done for three weeks. Besides, telephone follow-up was used for a few cases. After the follow-up, the FACT-An was completed by the participants in both groups.

The collected data were analyzed using SPSS software (version 22). First, frequency, percentage, mean, and standard deviation of the participants' scores were determined using descriptive statistics. Furthermore, the Shapiro-Wilk test was used to determine the data normality. The participants' mean scores in the two groups on the pretest and posttest were compared using the independent samples ttest. Moreover, the pretest and posttest scores of the participant in each group were compared using the pairedsamples t-test, and the frequency of the qualitative variables for the two groups was measured and compared using the chi-square test. All statistical tests were performed at the significance level of less than $0.05(\mathrm{P}<0.05)$.

\section{Results}

The results of data analysis showed that the mean scores for the age of the intervention and control groups were $46.20 \pm 9.76$ and $44.64 \pm 11.16$, respectively. Moreover, $64.4 \%$ of patients in the intervention group and $68.9 \%$ of patients in the control group were women. It was also indicated that $84.4 \%$ of the patients in the intervention group and $75.6 \%$ of the patients in the control group held a diploma and lower educational degrees. Besides, $62.2 \%$ and $46.7 \%$ of the intervention and control groups were not employed. Finally, the minimum and maximum albumin levels in both groups were 3.5 and $5.5 \mathrm{~g} / \mathrm{dL}$ (Table 1 ).

The results of the present study showed that the mean scores of hemoglobin in the cancer patients in the intervention group increased significantly after attending the nursing care program $(P<0.001)$. Similarly, the hemoglobin level increased in the patients in the control group after the mentioned period $(\mathrm{P}=0.01)$ (Table 2 ).

Moreover, 33.3\% of the patients in the intervention group lost their mild anemia and did not meet the criterion for anemia. However, only $6.7 \%$ of patients in the control group had no symptoms of anemia based on the hemoglobin index (Table 3).

It was also shown that the mean scores of fatigue in cancer patients in the intervention group decreased significantly after attending the nursing care program $(\mathrm{P}<$ 0.001). Likewise, the fatigue scores decreased in the patients in the control group after the mentioned period, but these changes were not significant $(P=0.73)$ (Table 4).

\section{Discussion}

The findings of the present study indicated the positive effect of the nursing care program supplemented with iron intake on blood parameters and fatigue of the patients undergoing chemotherapy. It was also shown that the hemoglobin of the patients in the intervention group increased after the nursing care program and the severity of their anemia decreased, confirming the effect of training strategies to increase iron absorption and intake. Similarly, Rezaee Seraji et al. showed that the hemoglobin concentration of people who did aerobic exercise increased compared to patients who did not perform the aerobic exercise (27). However, the authors emphasized aerobic exercise, and the changes in hemoglobin ratio were not very noticeable. In contrast, in the present study and physical activity, the patients received iron and proper nutrition training to increase iron absorption. The results proved the significant impact of the intervention.

One of the essential issues in treating patients with cancer is maintaining blood parameters to be within the normal range or close to it. When the patient refers to chemotherapy, nurses decide to perform or not to perform chemotherapy after reviewing the results of blood cell counts. Thus, the implementation of the treatment program depends entirely on the patient's blood test results. If the blood count decreases, the treatment program will be stopped, affecting the survival of patients. 


\begin{tabular}{|c|c|c|c|}
\hline \multirow{2}{*}{ Variable } & \multicolumn{2}{|c|}{ Groups } & \multirow{2}{*}{ P-Value } \\
\hline & Intervention & Control & \\
\hline Age & $46.20 \pm 9.76$ & $44.64 \pm 11.16$ & $0.48^{\mathrm{b}}$ \\
\hline Duration of illness & $1.71 \pm 0.92$ & $2.17 \pm 1.78$ & $0.12^{\mathrm{b}}$ \\
\hline Albumin & $4.21 \pm 0.73$ & $4.22 \pm 0.63$ & $0.96^{\mathrm{b}}$ \\
\hline \multicolumn{4}{|l|}{ Sex } \\
\hline Male & $16(35.6 \%)$ & $14(31.1 \%)$ & $0.65^{c}$ \\
\hline Female & $29(64.4 \%)$ & $31(68.9 \%)$ & \\
\hline \multicolumn{4}{|l|}{ Education } \\
\hline High school diploma and lower & $38(84.4 \%)$ & $34(75.6 \%)$ & $0.29^{c}$ \\
\hline Above the diploma & $7(15.6 \%)$ & $11(24.4 \%)$ & \\
\hline \multicolumn{4}{|l|}{ Occupation } \\
\hline Employed & $17(37.8 \%)$ & $24(53.3 \%)$ & $0.13^{c}$ \\
\hline Unemployed & $28(62.2 \%)$ & $21(46.7 \%)$ & \\
\hline
\end{tabular}

${ }^{\mathrm{a}}$ Values are expressed as mean $\pm \mathrm{SD}$ or No $(\%)$

${ }^{\mathrm{b}}$ Independent samples t-test.

${ }^{\mathrm{c}}$ Chi-square test.

Table 2. Comparison of Hemoglobin Level of Cancer Patients Undergoing Chemotherapy Before and After Iron-Based Nursing Care Program Between Intervention and Control Groups

\begin{tabular}{lccc}
\hline Group & Pretest Score & Posttest Score & Paired-Samples T-Test \\
\hline Intervention & $10.80 \pm 0.64$ & $11.84 \pm 0.58$ & $\mathrm{P}<0.001$ \\
Control & $10.98 \pm 0.76$ & $11.10 \pm 0.67$ & $\mathrm{P}=0.01$ \\
Independent samples t-test & $\mathrm{P}=0.23$ & $\mathrm{P}<0.0001$ & \\
\hline
\end{tabular}

Table 3. Comparison of Frequency Distribution of Anemia Severity in Cancer Patients Undergoing Chemotherapy After the Implementation of Nursing Care Program with Iron Intake Between the Intervention and Control Groups

\begin{tabular}{lcc}
\hline Anemia Severity & Intervention Group & Control Group \\
\hline Mild anemia $(\mathbf{H g}=\mathbf{1 0}-\mathbf{1 2})$ & $30(66.7 \%)$ & $42(93.3 \%)$ \\
No anemia $(\mathbf{H g}>\mathbf{~ 1 2})$ & $15(33.3 \%)$ & $3(6.7 \%)$ \\
\hline
\end{tabular}

Table 4. Comparison of Fatigue Scores of Cancer Patients Undergoing Chemotherapy Before and After Iron-Based Nursing Care Program Between Intervention and Control Groups

\begin{tabular}{lccc}
\hline Group & Pretest Score & Posttest Score & Paired-Samples T-Test \\
\hline Intervention group & $52.26 \pm 11.69$ & $29.37 \pm 9.56$ & $\mathrm{P}<0.001$ \\
Control group & $47.77 \pm 11.74$ & $47.55 \pm 11.22$ & $\mathrm{P}=0.73$ \\
Independent samples t-test & $\mathrm{P}=0.07$ & $\mathrm{P}<0.001$ & \\
\hline
\end{tabular}

In cancer control and treatment programs, the management of blood parameters, including the maintenance of hemoglobin within the normal range, is of great importance. Accordingly, the studies reviewed above indicated that anemia reduces the prognosis of cancer patients (29), influences the effectiveness of cancer treatments, and increases the likelihood of tumor cell resistance to treat- ment, which ultimately reduces the survival of the patient (30).

Studies have shown that a decrease in the level of erythrocytes and anemia is one of the most common and persistent problems in cancer patients (31), and its severity depends on the extent of the disease and the severity of treatment. It has been reported that anemia increases 
the risk of death by $65 \%$ in patients with various cancers (27). Kitano et al. showed that $84 \%$ of the patients developed anemia while undergoing chemotherapy and 72\% of the patients who had no anemia became anemic during chemotherapy (32). Bernardo's study also found that 73\% of patients developed anemia after starting chemotherapy (33). The prevalence of anemia was 50\% in solid cancers, as reported in Groopman's study and 35\% in Seshardi's study $(34,35)$. Probably the discrepancies observed in the frequencies reported in various studies are due to the type of study, the studied population, and the definition of anemia. The research sample in the present study included the patients with mild anemia or no symptoms of anemia, and thus the sample was different from those examined in previous studies. The results showed that patients who had attended the nursing care program supplemented with iron intake were less likely to have anemia during the treatment. It was also shown that the patients' hemoglobin level increased by one gram during the intervention, indicating that perhaps one of the factors that can affect the patients' fatigue is the hemoglobin level since the patients reported lower levels of fatigue after the increase in hemoglobin and iron intake in the form of the nursing care program that focused on doing exercise and daily activities, as was confirmed in other studies $(36,37)$. However, Cella et al. showed that the patients who had a 2-gram increase in hemoglobin reported less fatigue (38). These conflicting results can be attributed to the differences in the research population, nursing care program, and other factors. Nevertheless, the results show that improving anemia and hemoglobin levels can affect the severity of fatigue. Other studies also suggested that increasing hemoglobin levels reduces the patient's physical fatigue and improves the patient's physical function (38). On the other hand, anemia can lead to further problems such as fatigue $(38,39)$.

The present study suggested that a significant reduction in the fatigue scores of the patients in the intervention group indicated the effectiveness of the nursing care program. Studies have shown that fatigue is one of the most complex and common problems associated with cancer $(40,41)$. Fatigue in cancer patients may be caused by the disease process or related treatments called cancerinduced fatigue (42). Among the various cancer treatments, chemotherapy is the most common cause of fatigue in patients (43). Cancer-induced fatigue in many patients is so severe that, if left untreated, it disrupts and limits their daily activities (44). In the present study, a specific tool was used to measure anemia-related fatigue. This study was also different from other studies in terms of the procedure taken. Therefore, it is possible to discuss anemia-related fatigue with more confidence. As shown, the nursing care program with an emphasis on iron intake can significantly affect anemia-induced fatigue. Limited studies have been performed using this tool, which were primarily descriptive and did not use any intervention. For instance, Ghaffari et al. measured the relationship between the severity of fatigue and anemia and reported a moderate level of anemia-induced fatigue (24). In Timothy's study, $40 \%$ of patients suffered from moderate fatigue (36). Khozimeh et al. showed that the use of energy-saving strategies significantly reduced the patient's fatigue compared to the control group and the patients in the intervention group better managed their fatigue. Their main energy-saving strategy was to create a balance between the rest and activity times during fatigue induced by the disease (45). Another study by Abdollahi and Shujaedin suggested that the training protocol improved all aspects of the quality of life and increased physical performance in the intervention group compared to the control group (46), as was evident in the present study. Despite the effectiveness of exercise in controlling fatigue, studies have shown that the most frequently used strategies by patients were lying down (80.7\%) and worshiping (77.9\%) and the least frequently used strategies were exercise (5\%) and stretching movements (21.4\%). Accordingly, it can be argued that the first thing a person does in the face of an unpleasant feeling, such as fatigue, is to stop working and taking a rest. Furthermore, patients wrongly believe that their fatigue increases if they do things such as exercise and walking, and thus they do these activities less frequently (47). However, according to several studies, doing exercise plays an essential role in reducing the severity of fatigue (48-50).

The present study also suggested that the severity of fatigue was lower in the patients in the control group, but it was not significantly different from the fatigue level reported by the participants in the intervention group. One reason is that supplying iron reserves after taking iron supplements, which was effective in reducing fatigue in this group of patients. Besides, the participation of the patients in the control group in the treatment program and the emphasis on iron intake can be other reasons that have led to an increase in hemoglobin levels in the control group.

\subsection{Conclusion}

The present study showed that implementing nursing interventions for cancer patients receiving iron supplementation on proper iron intake can have a positive effect on iron-related blood parameters and patients' fatigue. Therefore, medical and nursing staff are recommended to pay special attention to these training interventions and also iron intake in their fatigue control and management programs. 


\section{Acknowledgments}

This article reports a master's thesis in intensive care nursing conducted in Zahedan School of Nursing and Midwifery. The authors would like to appreciate the research council of the Zahedan University of Medical Sciences, the head nurse, nurses, staff, and patients in the oncology ward of Khatam al-Anbia Hospital (PBUH) and the chemotherapy ward of Ali Ibn Abi Taleb Hospital (AS) for their help in collecting data.

\section{Footnotes}

Authors' Contribution: Zeinab Barahoui: data collection and manuscript composition. Fatemeh Kiani: study design and manuscript composition. Tayebelashkari: study design. Mojtaba Tasbandi: study design.

Conflict of Interests: The authors declare no conflict of interest.

Ethical Approval: This research was approved by the Zahedan University of Medical Sciences under the code of ethics IR.zaums.REC.1398.391 dated Feb. 2, 2020., and all ethical considerations in clinical studies were observed by the authors.

Funding/Support: This research project was financed by the Vice Chancellor for Research of Zahedan University of Medical Sciences.

Informed Consent: A written informed consent form was obtained from all patients.

\section{References}

1. Siegel RL, Miller KD, Jemal A. Cancer statistics, 2016. CA Cancer J Clin. 2016;66(1):7-30. doi: 10.3322/caac.21332. [PubMed: 26742998].

2. Esmail Nasab N, Moradi G, Zareie M, Ghaderi E, Gheytasi B. Survey of epidemilogic status and incidence rates of cancers in the patients above 15 years old in Kurdistan province. Sci J Kurdistan Univ Medical Sci. 2007;11(4):18-25.

3. Alilu L, Heydarzadeh L, Habibzadeh H, Rasouly J. The effect of peer education on gastrointestinal complications-based self-care inpatients with cancer referred to imam khomeini and omid hospitals of Urmia in 2018-2019. Nurs Midwifery J. 2020;12(1):947-54.

4. Hasanvand S, Ashktorab T, Jafari Z, Salmani N, Safariyan Z. Cancerrelated fatigue and its association with health-related quality of. $A d v$ Nurs Midwifery. 2015;24(85):21-30.

5. Faraji K, Neamatzade S, Saboori H. Radiation effect on the number of circulating blood cells in patients receiving radiation therapy in Tohid hospital of Sanandaj in 2014. Zanco J Med Sci. 2014;15(46):49-58.

6. Fani Pakdel A, Elyasi S, Kooshiar MM, Jannati Yazdan Abad M, Marouzi A, Asgarian M. Identification and analysis of adverse drug reactions associated with colorectal and gastric cancer chemotherapy in hospitalized patients. Med J Mashhad Univ Med Sci. 2018;61(2):921-30.

7. Bower JE. Cancer-related fatigue-mechanisms, risk factors, and treatments. Nat Rev Clin Oncol. 2014;11(10):597-609. doi: 10.1038/nrclinonc.2014.127. [PubMed: 25113839]. [PubMed Central: PMC4664449].
8. Berger AM, Mooney K, Alvarez-Perez A, Breitbart WS, Carpenter KM, Cella D, et al. Cancer-related fatigue, version 2.2015. J Natl Compr Canc Netw. 2015;13(8):1012-39. doi: 10.6004/jnccn.2015.0122. [PubMed: 26285247]. [PubMed Central: PMC5499710].

9. Moghaddam Tabrizi F, Alizadeh S, Gozali N. The effect of energy conservation strategies on cancer-related fatigue during breast cancer chemotherapy in Urmia. Nurs Midwifery J. 2017;14(10):830-6.

10. Ahmadi Gharagazloo N, Rahimian Booger I, Asadi J, Vojdani R. Improvement of cancer related fatigue in breast and colorectal cancer patients taking part in mindfulness based stress reduction training program. Armaghane danesh. 2019;24(3):306-18.

11. Carpenito LJ. Nursing care plans: Transitional patient $\&$ family centered care. Lippincott Williams \& Wilkins; 2013. 76 p.

12. Wells JN, Fedric T. Helping patients manage cancer-related fatigue. Home Healthc Nurse. 2001;19(8):486-93. quiz 493-4. doi: 10.1097/00004045-200108000-00010. [PubMed: 11982185].

13. Headley JA, Ownby KK, John LD. The effect of seated exercise on fatigue and quality of life in women with advanced breast cancer. Oncol Nurs Forum. 2004;31(5):977-83. doi: 10.1188/04.ONF.977-983. [PubMed: 15378098].

14. Mohandas H, Jaganathan SK, Mani MP, Ayyar M, Rohini Thevi GV. Cancer-related fatigue treatment: An overview. J Cancer Res Ther 2017;13(6):916-29. doi:10.4103/jcrt.JCRT_50_17. [PubMed: 29237952].

15. Busti F, Marchi G, Ugolini S, Castagna A, Girelli D. Anemia and iron deficiency in cancer patients: Role of iron replacement therapy. Phar maceuticals (Basel). 2018;11(4). doi: 10.3390/ph11040094. [PubMed: 30274354]. [PubMed Central: PMC6315653].

16. Sh. Zeighami Mohamadi; H. Esmaili; M.M. Kooshyar. Intensity of fatigue in cancer patients before and after 3 cycles chemotherapy. Avicenna If Nurs Midwifery Care. 2007;15(2):12-22.

17. Visweshwar N, Jaglal M, Sokol L, Zuckerman K. Chemotherapy-related anemia. Ann Hematol. 2018;97(2):375-6. doi: 10.1007/s00277-017-3155-y. [PubMed: 29103107].

18. Mhaskar R, Wao H, Miladinovic B, Kumar A, Djulbegovic B. The role of iron in the management of chemotherapy-induced anemia in cancer patients receiving erythropoiesis-stimulating agents. Cochrane Database Syst Rev. 2016;2. CD009624. doi: 10.1002/14651858.CD009624.pub2. [PubMed: 26845108].

19. Sh.Zeighami Mohammedi; P. Hushmand; F. Jafari; M.M. Kooshyar. The relationship of anemia with severity of fatigue and quality of life in cancer patient undergoing chemotherapy. Avicenna J Nurs Midwifery Care. 2008;16(2):43-53.

20. Cella D. The functional assessment of cancer therapy-anemia (FACTAn) scale: A new tool for the assessment of outcomes in cancer anemia and fatigue. Semin Hematol. 1997;34(3 Suppl 2):13-9. [PubMed: 9253779].

21. Holzner B, Kemmler G, Greil R, Kopp M, Zeimet A, Raderer M, et al. The impact of hemoglobin levels on fatigue and quality of life in cancer patients. Ann Oncol. 2002;13(6):965-73. doi: 10.1093/annonc/mdfi22. [PubMed: 12123343].

22. Ludwig H, Evstatiev R, Kornek G, Aapro M, Bauernhofer T, BuxhoferAusch V, et al. Iron metabolism and iron supplementation in cancer patients. Wien Klin Wochenschr. 2015;127(23-24):907-19. doi: 10.1007/s00508-015-0842-3. [PubMed: 26373748]. [PubMed Central: PMC4679104].

23. Manz DH, Blanchette NL, Paul BT, Torti FM, Torti SV. Iron and cancer: recent insights. Ann N Y Acad Sci. 2016;1368(1):149-61. doi: 10.1111/nyas.13008. [PubMed: 26890363]. [PubMed Central: PMC4870095]

24. Ghaffari F, Fotokian Z, Karimi M, Keihanian S, Karimi H. The relationship between anemia-related fatigue and quality of life in canceric patients. J Inflamm Dis. 2009;13(1):34-41.

25. Godino C, Jodar L, Duran A, Martinez I, Schiaffino A. Nursing education as an intervention to decrease fatigue perception in oncology patients. Eur J Oncol Nurs. 2006;10(2):150-5. doi 10.1016/j.ejon.2005.03.004. [PubMed:16618589]. 
26. Shobeyri F, Nikravesh A, Masoumi SZ, HeydariMoghadam R, Karami $\mathrm{M}$, Badafreh M. Effect of exercise counseling on functional scales quality of life in women with breast cancer. J Ed Community Health. 2015;2(1):1-9.

27. Rezaee Seraji B, Ravasi AA, Hajifathali A, Soori R, Mahdizadeh M, Amini $M$. The effects of aerobic exercise on erythrocyte indices in cancer patients after autologous hematopoietic stem cell transplantation. Sci Iran Blood Transfus Org. 2012;9(3):251-7.

28. Ramezanpour MR, Kazemi M. Effects of aerobic training along with iron supplementation on the hemoglobin, red blood cells, hematocrit, serum ferritin, transferrin and iron in young girls. Koomesh. 2012;13(2).

29. Caro J, Salas M, Ward A, Goss G. Anemia as an independent prognostic factor for survival in patients with cancer. Cancer. 2001;91(12):2214-21. doi:10.1002/1097-0142(20010615)91:12<2214::aid-cncr1251>3.0.co;2-p.

30. Vaupel P, Mayer A. Hypoxia and anemia: effects on tumor biology and treatment resistance. Transfusion clinique et biologique. 2005;12(1):510 .

31. Lebrun F, Klastersky J, Levacq D, Wissam Y, Paesmans M. Intravenous iron therapy for anemic cancer patients: a review of recently published clinical studies. Support Care Cancer. 2017;25(7):2313-9. doi: 10.1007/s00520-017-3672-1. [PubMed: 28386789].

32. Kitano T, Tada H, Nishimura T, Teramukai S, Kanai M, Nishimura T, et al. Prevalence and incidence of anemia in Japanese cancer patients receiving outpatient chemotherapy. Int J Hematol. 2007;86(1):37-41. doi 10.1532/IJH97.07040. [PubMed: 17675265].

33. Bernardo G, Aglietta M, Amadori D, Beccaglia P, Belli G, Bianco $\mathrm{R}$, et al. [Prevalence of anemia in oncologic patients treated with chemotherapy. Italian survey at the national level]. Recenti Prog Med. 2001;92(10):580-8. [PubMed: 11695301].

34. Groopman JE, Itri LM. Chemotherapy-induced anemia in adults: incidence and treatment. J Natl Cancer Inst. 1999;91(19):1616-34. doi: 10.1093/jnci/91.19.1616. [PubMed: 10511589].

35. Seshadri T, Prince HM, Bell DR, Coughlin PB, James PP, Richardson GE, et al. The australian cancer anaemia survey: A snapshot of anaemia in adult patients with cancer. Med J Aust. 2005;182(9):453-7. [PubMed: 15865588].

36. Littlewood TJ, Kallich JD, San Miguel J, Hendricks L, Hedenus M. Efficacy of darbepoetin alfa in alleviating fatigue and the effect of fatigue on quality of life in anemic patients with lymphoproliferative malignancies. J Pain Symptom Manage. 2006;31(4):317-25. doi: 10.1016/j.jpainsymman.2005.08.013. [PubMed:16632079].

37. Cortesi E, Gascon P, Henry D, Littlewood T, Milroy R, Pronzato P, et al. Standard of care for cancer-related anemia: improving hemoglobin levels and quality of life. Oncology. 2005;68 Suppl 1:22-32. doi: 10.1159/000083130. [PubMed: 15855813].

38. Cella D, Kallich J, McDermott A, Xu X. The longitudinal relationship of hemoglobin, fatigue and quality of life in anemic cancer patients: re- sults from five randomized clinical trials. Ann Oncol. 2004;15(6):97986. doi:10.1093/annonc/mdh235. [PubMed:15151958].

39. Ajam M, Aghayari A, Salek R, Haghverdian S, Gheitasi M. Effect of 12 weeks aerobic exercise training on some characteristics of immune system and general health of women with breast cancer. Res Sport Med Technol. 2014;12(7):41-54.

40. Mardanian Dehkordi L, kahangi LS, Babashahi M. Evaluation of fatigue and its related factors in cancer patients undergoing chemotherapy. I Health Care. 2018;20(2):156-64. doi: 10.29252/jhc.20.2.156

41. Safaee A, Tabatabaee SH, Moghimi-Dehkordi B, Zeighami B. Cancerrelated fatigue in breast cancer patients under chemotherapy. Koomesh J. 2010;11(4):317-22.

42. Chehrehgosha M, Dastourpour M, Sanagu A, Mohamadi A. Cancerrelated fatigue and its relationship with demographic and clinical characteristics. Jorjani Biomed J. 2013;1(2):24-31.

43. Farajollahi M, Alikhani M, Farmani F, Hosseini F. Fatigue in cancer patients receiving chemotherapy. Iran J Nurs. 2004;16(36):47-52.

44. Rad M, Borzoee F, Mohebbi M. The effect of humor therapy on fatigue severity and quality of life in breast cancer patients undergoing external radiation therapy. J Adv Med Biomed Res. 2016;24(103):102-14.

45. Khozimeh FS, Navidian A, Sasanpour P, Kiani F. The Effect of Training on Energy Conservation Strategies, Fatigue, and Self-Caring among Women with Breast Cancer Undergoing Chemotherapy in Zahedan Medical Sciences Hospitals in 2018-2019. J Evol Med Dental Sci. 2019;8(49):3661-6. doi: 10.14260/jemds/2019/792.

46. Abdollahi M, Shujaedin S. Effect of six-weeks of mixed exercises on improving quality of life and physical performance after chemotherapy in patients with colon cance. SciJRehab Med.2020;8(4):218-26. doi: 10.22037/jrm.2019.111441.1997.

47. Faragollahi M. Strategies used by patients receiving chemotherapy to relieve fatigue. Iran J Nurs. 2004;17(38):58-64.

48. Gheyasi F, Baraz S, Malehi A, Ahmadzadeh A, Salehi R, Vaismoradi M. Effect of the walking exercise program on cancer-related fatigue in patients with acute myeloid leukemia undergoing chemotherapy. Asian Pac J Cancer Prev. 2019;20(6):1661-6. doi: 10.31557/APJCP.2019.20.6.1661. [PubMed: 31244285]. [PubMed Central: PMC7021614].

49. Andersen C, Rorth M, Ejlertsen B, Stage M, Moller T, Midtgaard J, et al. The effects of a six-week supervised multimodal exercise intervention during chemotherapy on cancer-related fatigue. Eur J Oncol Nurs. 2013;17(3):331-9. doi: 10.1016/j.ejon.2012.09.003. [PubMed: 23084254].

50. Kelley GA, Kelley KS. Aerobic exercise and cancer-related fatigue in adults: A reexamination using the ivhet model for metaanalysis. Cancer Epidemiol Biomarkers Prev. 2017;26(2):281-3. doi: 10.1158/1055-9965.EPI-16-0885. [PubMed: 27908923]. [PubMed Central: PMC5296275] 